OPEN ACCESS

Edited by:

Alessandro Granito,

University of Bologna, Italy

Reviewed by:

Malcolm Koo,

Tzu Chi University of Science and

Technology, Taiwan

Wen-chi Chen

China Medical University

Hospital, Taiwan

*Correspondence:

Chia-Hung Kao

d10040@mail.cmuh.org.tw;

dr.kaochiahung@gmail.com

Specialty section:

This article was submitted to

Gastroenterology,

a section of the journal

Frontiers in Medicine

Received: 03 March 2021

Accepted: 14 April 2021

Published: 12 May 2021

Citation:

Wang T-Y, Lai H-C, Chen H-H, Wang M-L, Hsieh M-C, Chang C-T,

Chen R-H, Ho C-W, Hung Y-C,

Tseng J-Y, Lin C-L and Kao C-H (2021) Pyogenic Liver Abscess Risk in

Patients With Newly Diagnosed Type 2 Diabetes Mellitus: A Nationwide,

Population-Based Cohort Study. Front. Med. 8:675345.

doi: 10.3389/fmed.2021.675345

\section{Pyogenic Liver Abscess Risk in Patients With Newly Diagnosed Type 2 Diabetes Mellitus: A Nationwide, Population-Based Cohort Study}

\author{
Tzu-Yuan Wang ${ }^{1,2}$, Hsueh-Chou Lai ${ }^{3}$, Hsin-Hung Chen ${ }^{4,5,6}$, Mei-Lin Wang ${ }^{7}$, \\ Ming-Chia Hsieh ${ }^{1}$, Chwen-Tzuei Chang ${ }^{1}$, Rong-Hsing Chen ${ }^{1}$, Chun-Wei Ho ${ }^{1}$, \\ Yi-Chin Hung ${ }^{1}$, Juei-Yu Tseng ${ }^{1}$, Cheng-Li Lin ${ }^{8,9}$ and Chia-Hung Kao ${ }^{10,11,12,13 *}$
}

${ }^{1}$ Intelligent Diabetes Metabolism and Exercise Center, Department of Internal Medicine, China Medical University Hospital, Taichung, Taiwan, ${ }^{2}$ School of Medicine, China Medical University, Taichung, Taiwan, ${ }^{3}$ Division of Hepato-Gastroenterology, Department of Internal Medicine, China Medical University Hospital, Taichung, Taiwan, ${ }^{4}$ Division of Endocrinology and Metabolism, Department of Internal Medicine, Asia University Hospital, Taichung, Taiwan, ${ }^{5}$ School of Medicine, Chung Shan Medical University, Taichung, Taiwan, ${ }^{6}$ Chung Sheng Clinic, Nantou, Taiwan, ${ }^{7}$ Department of Nursing, Hung Kuang University, Taichung, Taiwan, ${ }^{8}$ Management Office for Health Data, China Medical University Hospital, Taichung, Taiwan, ${ }^{9}$ College of Medicine, China Medical University, Taichung, Taiwan, ${ }^{10}$ Graduate Institute of Biomedical Sciences Science, School of Medicine, College of Medicine, China Medical University, Taichung, Taiwan, "11 Department of Nuclear Medicine and Positron Emission Tomography (PET) Center, China Medical University Hospital, Taichung, Taiwan, ${ }^{12}$ Department of Bioinformatics and Medical Engineering, Asia University, Taichung, Taiwan, ${ }^{13}$ Center of Augmented Intelligence in Healthcare, China Medical University Hospital, Taichung, Taiwan

Background: To date, no comprehensive epidemiological study exists on pyogenic liver abscess (PLA) risk in patients with newly diagnosed type 2 diabetes mellitus (T2DM) worldwide.

Methods: We conducted a retrospective cohort study by using data from Taiwan National Health Insurance Research Database (NHIRD) to examine the association between newly diagnosed T2DM and PLA. The T2DM cohort included patients newly diagnosed as having T2DM (ICD-9-CM:250) from 2000 to 2009, with follow-up until December 31, 2011. The comparison cohort was then recruited through 1:4 random frequency matching with the T2DM cohort. Finally, the adjusted hazard ratios for PLA were compared between the T2DM and comparison cohorts, which included 44,728 patients with T2DM and 178,912 patients without DM respectively.

Results: In T2DM cohort, 166 patients were diagnosed as having PLA (incidence rate $=5.87$ per 10,000 person-years) and in comparison cohort, 238 patients were diagnosed as having PLA (incidence rate $=2.06$ per 10,000 person-years). The T2DM cohort exhibited higher PLA risk than did the comparison cohort (hazard ratio $=2.83$, $95 \%$ confidence interval $=2.32-3.46)$. Furthermore, the adjusted hazard ratio for PLA risk in T2DM cohort was the highest in those who were younger, man and with duration of DM <2 years. In the T2DM cohort, the most common PLA causative agent was Klebsiella pneumonia (KP). In addition, PLA risk was high in T2DM patients with gallstone and cholecystitis. Compared with comparison cohort, patients with T2DM prescribed acarbose has a lower PLA risk, however glyburide significantly increased PLA risk in T2DM cohort. 
Conclusion: In patients with newly diagnosed T2DM, PLA risk was high and acarbose might reduce PLA risk.

Keywords: pyogenic liver abscess, newly diagnosed type 2 diabetic mellitus, Klebsiella pneumonia, acarbose, National Health Insurance Research Database

\section{WHAT IS ALREADY KNOWN ABOUT THIS TOPIC?}

To date, no comprehensive epidemiological study exists on pyogenic liver abscess (PLA) risk in patients with newly diagnosed type 2 diabetes mellitus (T2DM) worldwide.

\section{WHAT DOES THIS ARTICLE ADD?}

In patients with newly diagnosed T2DM, PLA risk was high and acarbose might reduce PLA risk.

\section{INTRODUCTION}

Pyogenic liver abscess (PLA) is a serious infectious and lifethreatening disease with a low but gradually increasing annual incidence rate. The incidence rate was only 1.0 per 100,000 person-years from 1997 to 2002 in Denmark (1) and 2.3 per 100,000 person-years from 1999 to 2003 in Canada (2). However, PLA incidence rate is much higher in Taiwan. A nationwide analysis from the National Health Insurance Research Database (NHIRD) data from 2000 through 2011 demonstrated that the annual incidence of PLA for all ages groups increased gradually from 10.83 per 100,000 person-years in 2000 to 15.45 per 100,000 person-years in 2011 (3). Moreover, during 2000-2011, the average annual incidence was 13.52 per 100,000 person-years. PLA has become a health problem in the Taiwanese society.

PLAs often results from the complications of biliary tract infection, and globally, the most common PLA pathogen is Escherichia coli (E. coli) (4). However, accumulating evidence in Taiwan has shown a direct relationship between PLA and Klebsiella pneumoniae (KP) in patients with Diabetes Mellitus (DM) without biliary tract infection (5-7). Over 1981-1993, the medical records of 146 adults treated for PLA in the hospitals in Taipei were retrospectively investigated. Of all PLA cases, 78\% were due to KP, and more patients with PLA causing by KP were observed to exhibit DM than did patients with non- KP PLA (66 vs. 19\%) (5). In another study, 182 patients with PLAs treated at Kaohsiung Veterans General Hospital, Taiwan from 1990 to 1996 were enrolled. Of these patients, $87.9 \%$ had PLAs caused by KP. In addition, patients with PLA causes by KP exhibited higher DM and glucose intolerance incidence rate than did patients with non-KP PLA (75 vs. 4.5\%) (6). Furthermore, a study in Taiwan demonstrated that of 1,522 adults with PLA, 35.3\% had DM, $10.8 \%$ had complications, $15.4 \%$ received mechanical ventilation and $23.7 \%$ were provided intensive care (3). These data suggest that PLA caused by KP is closely associated with DM. Furthermore, several aspects are believed to be affected in patients with DM such as polymorphonuclear leukocyte function inhibition, leukocyte adherence, chemotaxis, phagocytosis and impairment of antioxidant systems involved in bactericidal activity (7).

To date, no comprehensive epidemiological study exists for PLA risk in patients with newly diagnosed T2DM worldwide. Thus, we conducted a large retrospective cohort study by using the health care service file from the NHIRD to examine the association between newly diagnosed T2DM and PLA risk. We compared the clinical manifestations, clinical outcomes and drug treatments recorded for patients with PLA in the DM and nonDM groups.

\section{PATIENTS AND METHODS}

\section{Data Sources}

We used the Longitudinal Health Insurance Database (LHID) for this study. The LHID is a subset of NHIRD which contains all claims data from the Taiwan National Health Insurance (NHI) beneficiaries (including nearly 23 million Taiwan residents). The NHI system of Taiwan was established in 1995 and covers more than $99.6 \%$ of the Taiwanese population; this system's claims data are released as the NHIRD (8). The LHID was contains 1996-2000 data of random 1 million insured people randomly selected from NHI beneficiaries. According to the Taiwan government report, the age and sex distribution are not different for the LHID and NHIRD. The LHID features claims data including a registry for beneficiaries, records of clinic care and hospital care, drug prescriptions and other medical services and these data are renewed annually. The disease record system of clinic and hospital care is based on International Classification of Diseases, Ninth Revision, Clinical Modification (ICD-9-CM). Before the data are releasing for research, the Taiwan government implements privacy protection for insured individuals, removes the original identification numbers and provides an encoded serial numbers for insured people to link their claims data. Furthermore, this study was approved by the Ethics Review Board of China Medical University (CMUH104REC2-115-AR4).

\section{Study Population}

We extracted a T2DM and comparison cohorts from the aforementioned databases and compared PLA risks between them. In the T2DM cohort, patients newly diagnosed as having T2DM (ICD-9-CM 250) from 2000 to 2009 at two outpatient records within 1 year were enrolled from the LHID. We used date of initial T2DM diagnosis as the index date. In the comparison, individuals without a history of DM from LHID were randomly 1:4 frequency-matched with the T2DM cohort, the matching was based on the age of cohort entry (per 5 years) and sex. The index date for the patients in the comparison cohort was 


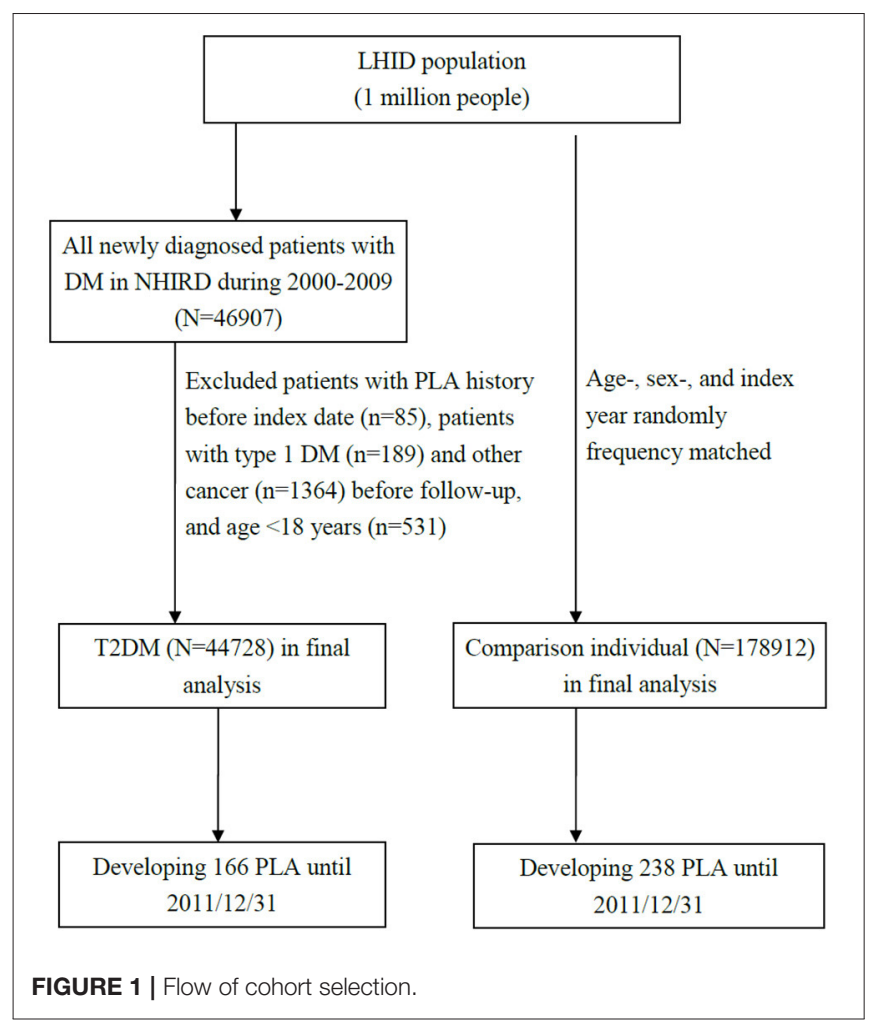

created by matching the year of the index date of patients in the T2DM cohort. We excluded participants age $<18$ years or type 1 DM, PLA or cancer before the index date. The study outcome of the interest was the occurrence of PLAs (ICD-9-CM 572.0) at inpatient record. For both cohorts follow-up commenced at index date and was terminated when the individual withdrew insurance, when PLA occurred or on December 31, 2011. Figure 1 depicts the flow for cohort selection.

\section{Criteria and Definitions}

Age, sex and PLA-associated comorbidities were considered confounding factors in this study. The PLA-associated comorbidity was defined as individuals with a comorbidity diagnosis history before the index date and comorbidities included gallstone (ICD-9-CM 574), cholecystitis (ICD-9-CM 575.0 and 575.10) and cholangitis (ICD-9-CM 576.1).

This study also investigated the effect of DM medication on PLA risk in patients with T2DM. Patients administered DM medications during the observation time were grouped according to the type of medication used. The DM medications included metformin, glyburide, glimepiride, gliclazide, glipizide, thiazolidinedione (TZD; including rosiglitazone and pioglitazone), dipeptidyl peptidase-4 inhibitor (DPP4i) and acarbose.

The microorganisms were identified on the same date as the first diagnosis of PLA during hospitalization. The microorganisms included Staphylococcus (ICD-9-CM 038.0 and 041.1), E.coli (ICD-9-CM 038.42 and 041.4), Streptococcus (ICD9-CM 038.0 and 041.0), Pneumococcus (ICD-9-CM 038.1 and
TABLE 1 | Baseline demographic status and comorbidity for comparison and T2DM cohorts.

\begin{tabular}{|c|c|c|c|}
\hline Variable & $\begin{array}{l}\text { Comparison cohort } \\
N=178,912 \text { (\%) }\end{array}$ & $\begin{array}{l}\text { T2DM cohort } \\
N=44,728(\%)\end{array}$ & $p$-value \\
\hline Age, years (SD) ${ }^{\star}$ & $55.5(14.1)$ & $55.6(13.9)$ & 0.1204 \\
\hline$<40$ & 23,404 (13.1) & $5,851(13.1)$ & \\
\hline $40-59$ & 90,192 (50.4) & $22,548(50.4)$ & \\
\hline $60-79$ & 57,776 (32.3) & 14,444 (32.3) & \\
\hline$\geqq 80$ & $7,540(4.2)$ & $1,885(4.2)$ & \\
\hline Sex & & & $>0.99$ \\
\hline Female & $82,008(45.8)$ & 20,502 (45.8) & \\
\hline Male & $96,904(54.2)$ & $24,226(54.2)$ & \\
\hline \multicolumn{4}{|l|}{ Comorbidity } \\
\hline Gallstone & $5,625(3.1)$ & 1,684 (3.8) & $<0.0001$ \\
\hline Cholecystitis & $797(0.4)$ & $261(0.6)$ & 0.0001 \\
\hline Cholangitis & $585(0.3)$ & $163(0.4)$ & 0.2198 \\
\hline \multicolumn{4}{|c|}{ Anti DM medication } \\
\hline Metformin & & $27,030(60.4)$ & \\
\hline Glyburide & & $10,595(23.7)$ & \\
\hline Glimepiride & & $16,338(36.5)$ & \\
\hline Gliclazide & & 13,939 (31.2) & \\
\hline Glipizide & & 7,071 (15.8) & \\
\hline TZD & & 7,354 (16.4) & \\
\hline DPP4i & & $4,545(10.2)$ & \\
\hline Acarbose & & 7,578 (16.9) & \\
\hline Others & & 6,177 (13.8) & \\
\hline
\end{tabular}

${ }^{*} t$-test.

TZD, thiazolidinedione; DPP4i, Dipeptidyl peptidase-4 inhibitor.

041.2), KP (ICD-9-CM 041.3), Proteus (ICD-9-CM 041.6), gramnegative bacteria (ICD-9-CM 038.40, 038.49, and 041.85), and other/unspecified bacteria (ICD-9-CM 038.8, 038.9, and 041).

\section{Statistical Analysis}

The study population characteristics are presented as the means and standard deviation (SDs) for age and as numbers and percentages for sex, comorbidity and DM. In each group, PLA incidence rate was calculated as number of patients divided by the sum of follow-up duration (per 10,000 person-years). The PLA cumulative incidence curve was measured for the T2DM cohort and comparison cohorts by using Kaplan-Meier method. We assessed the difference between the curves for the two study cohorts by using log-rank test. PLA risk in the T2DM and comparison cohorts are presented as hazard ratios (HRs) and $95 \%$ confidence intervals (CIs) which were estimated by using a Cox proportional hazard regression model. We also analyzed the PLA risk in individuals with and without DM stratified by age, sex, comorbidities and follow-up duration. All analyses were performed on SAS (version 9.4; SAS Institute, Cary, NC, USA) and the cumulative curve was plotted using R (R Foundation for Statistical Computing, Vienna, Austria). To assess the distribution difference for the individuals with and without DM, we used the two sample t- test for age and the chisquare test for sex and comorbidity. We calculated the incidence 


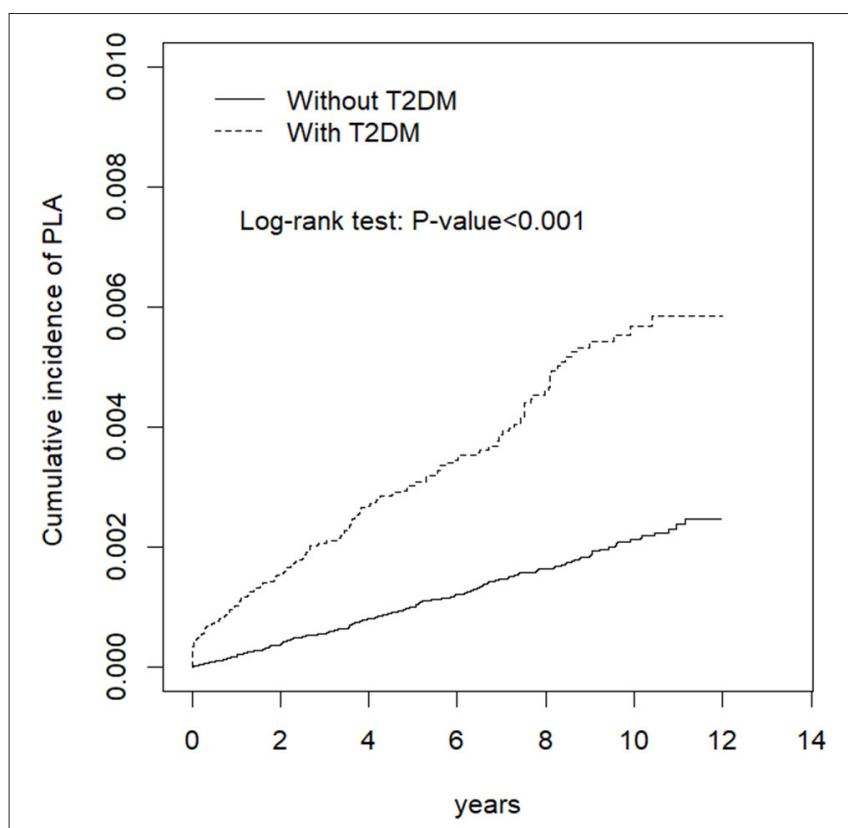

FIGURE 2 | Cumulative incidence of PLA in the T2DM and comparison cohort.

density of PLA for each study cohort, with statistical significance defined as a two-tailed $p$ of $<0.05$.

\section{RESULTS}

We enrolled a cohort of 44,728 patients with T2DM and 178,912 age and sex matched individuals (Table 1). Except for cholangitis, the rates of gallstone and cholecystitis occurrences in patients with T2DM were significantly higher than the proportions in comparison patients $(\mathrm{p}<0.05)$. In patients with T2DM, the most used medications were metformin $(60.4 \%)$, glimepiride $(36.5 \%)$ and gliclazide (31.2\%).

PLA incidence rate was 2.06 and 5.87 per 10,000 person-years in the comparison and T2DM cohorts, respectively (Table 2). Figure 2 also shows cumulative incidence was significantly higher in the T2DM cohort than in the comparison cohort $(\mathrm{P}<0.0001$, log-rank test). After adjustments for age, sex, gallstone occurrences, cholecystitis occurrences and cholangitis occurrences, the T2DM cohort has a higher PLA risk than did the comparison cohort $(\mathrm{HR}=2.83,95 \% \mathrm{CI}=2.32-3.46)$. Moreover, PLA risk was higher in older patients, $\operatorname{man}(\mathrm{HR}=1.69$, $95 \% \mathrm{CI}=1.38-2.07)$, patient with gallstone $(\mathrm{HR}=2.63,95 \%$ $\mathrm{CI}=1.81-3.80)$ and patients with cholangitis $(\mathrm{HR}=4.62,95 \%$ $\mathrm{CI}=2.44-8.74)$.

In patients with T2DM, Table 3 presents the PLA risk associated with various DM medications. After adjustments for sex, age, comorbidity, and DM medication uses, patients with DM administered glyburide were found to exhibit a significantly increased PLA risk compared with patients not administered glyburide $(\mathrm{HR}=1.84,95 \% \mathrm{CI}=1.27-2.66)$. Compared with those not administered acarbose, administered acarbose had lower PLA risk $(\mathrm{HR}=0.51,95 \% \mathrm{CI}=0.29-0.90)$.
Table 4 presents the results of our demographic factor and comorbidity-stratified analysis for PLA risk in our cohort. Except for those $\geq 80$ years, all patients with DM exhibited an increased HR for PLA risk being particularly high in those aged $<40$ years $(\mathrm{HR}=7.55,95 \% \mathrm{CI}=3.20-17.8)$. Compared with the comparison cohort. T2DM was significantly associated with a higher PLA risk in both women $(\mathrm{HR}=2.43,95 \% \mathrm{CI}=1.73-3.40)$ and men $(\mathrm{HR}=3.10,95 \% \mathrm{CI}=2.43-3.97)$. Moreover, PLA risk was higher in patients with T2DM without comorbidities than in the comparison cohort without comorbidities.

In Table 5, the PLA risk is compared between the T2DM and comparison cohorts for various follow-up durations. Compared with the individual without T2DM, PLA risk was the highest $<2$ years after $\mathrm{DM}$ diagnosis $(\mathrm{HR}=4.02,95 \% \mathrm{CI}=2.87-5.63)$. PLA risk was nearly 2-fold higher $2-4$ years $(\mathrm{HR}=2.74,95 \%$ $\mathrm{CI}=1.85-4.06)$ and $\geqq 4$ years $(\mathrm{HR}=2.13,95 \% \mathrm{CI}=1.55-2.95)$ after DM diagnosis.

Of all PLA patients with PLAs, $80.7 \%$ with T2DM and $84.9 \%$ without T2DM demonstrated positive microorganism test results (Table 6). KP was prevalent microorganisms in both the T2DM (30.7\%) and comparison (27.8\%) cohorts.

\section{DISCUSSION}

Our report is the first retrospective population-based cohort study that use the NHIRD in Taiwan to investigate the relationship between newly diagnosed T2DM and subsequent PLA risk. The analyzed data confirmed that patients with newly diagnosed T2DM had a higher PLA risk than did those without T2DM. Here, the T2DM cohort included patients newly diagnosed as having T2DM from 2000 to 2009, who were then followed until 2011. The PLA incidence rate reached 5.87 and 2.06 per 10,000 person-years in the T2DM and comparison cohorts, respectively. Patients with newly diagnosed T2DM had a 2.83-fold higher PLA risk than did patients with PLA but without T2DM. Increased PLA risk in patients with DM was also reported in Danish large nationwide case-control study (9), according to which individuals with DM had a 3.6-fold increased PLA risk. Moreover, in Canada, an epidemiological study showed that the relative risk of PLA to be 11.1 in patients with DM (2). The potential reasons underlying this observation are as follows: First, patients with diabetes have a higher risk of susceptibility to various common or serious infections $(10,11)$.

Although the pathophysiology of infections related to DM remains unclear, they may be caused by the hyperglycemic environment which results in immune dysfunction. Defects in leukocyte function, such as adherence, chemotaxis and phagocytosis have been reported in patients with DM (12). Second, complement deficiencies such as C3 or C4 deficiencies which are potentially associated with decreased cytokine response and reduced immobilization of polymorphonuclear leukocyte have been observed in many patients with DM (13), and the antioxidant systems related to antibacterial activity may also be damaged. KP is a major causative agent of PLA in Taiwan, particularly in patients with DM $(14,15)$. 
TABLE 2 | HRs and adjusted HRs for PLA in the study cohort.

\begin{tabular}{|c|c|c|c|c|c|}
\hline Variable & Event & PYs & Rate & HR (95\% Cl) & $\operatorname{aHR}^{\dagger}(95 \% \mathrm{Cl})$ \\
\hline \multicolumn{6}{|l|}{ DM } \\
\hline No & 238 & $1,152,686$ & 2.06 & Ref & Ref \\
\hline Yes & 166 & 282,924 & 5.87 & $2.84(2.33-3.46)$ & $2.83(2.32-3.46)$ \\
\hline \multicolumn{6}{|c|}{ Age group } \\
\hline$<40$ & 23 & 197,460 & 1.16 & Ref & Ref \\
\hline$\geqq 80$ & 24 & 40,528 & 5.92 & $5.01(2.83-8.89)$ & $4.55(2.55-8.10)$ \\
\hline \multicolumn{6}{|l|}{ Sex } \\
\hline Female & 145 & 675,211 & 2.15 & Ref & Ref \\
\hline Male & 259 & 760,399 & 3.41 & $1.58(1.29-1.94)$ & $1.69(1.38-2.07)$ \\
\hline \multicolumn{6}{|c|}{ Gallstone } \\
\hline No & 400 & $1,429,802$ & 2.80 & Ref & Ref \\
\hline Yes & 4 & 5,808 & 6.89 & $2.45(0.91-6.55)$ & $0.77(0.28-2.14)$ \\
\hline \multicolumn{6}{|c|}{ Cholangitis } \\
\hline No & 391 & $1,431,849$ & 2.73 & Ref & Ref \\
\hline Yes & 13 & 3,761 & 34.56 & $12.54(7.21-21.79)$ & $4.62(2.44-8.74)$ \\
\hline
\end{tabular}

${ }^{\dagger}$ Model adjusted for age, sex, gallstone, cholecystitis, and cholangitis.

PYs, person-years; Rate, incidence rate, per 10,000 person-years.

A Taiwanese study on the impact of glycemic control on the characteristics of PLA caused by KP in patients with DM reported that patients with uncontrolled glycemia tended to be younger, and have higher PLA rates of cryptogenic invasive PLA, caused by KP and metastatic infection than did those with good glycemic control (7). In our study, we found that the adjusted HR for PLA risk in the DM cohort was the highest in the youngest age group compared with the comparison cohort, and that men had a greater adjusted HR than women did, as was also reported in Western countries and Taiwan $(2,7,9)$. Several features that apply to younger patients with T2DM may contribute to the poor glycemic control. Younger patients with T2DM are more likely to be busy with work and less motivated attend to their condition. Smoking, obesity, life style factors, lack of confidence in doctors, and poor compliance with medications are also more common among younger peoples (16). Consistent with the findings of reports elsewhere, the most common microorganism in patients with $\mathrm{T} 2 \mathrm{DM}$ was $\mathrm{KP}(7,14,15)$, and a close association with PLA was observed in our present study. The pathophysiology of KP infection associated with DM is incompletely understood. However, the susceptibility of infection appears to be strongly associated with hyperglycemia Decreased mobilization of polymorphonuclear leukocytes, chemotaxis, and phagocytic activity may occur during hyperglycemia (17-19).

Possible sources reported for PLAs include biliary tract infections (such as cholangitis and cholecystitis), followed by portal venous disease, contiguous spread and hematogenous spread $(20,21)$. One study also showed that some infections almost always affect only patients with DM, such as gangrenous cholecystitis (22). Cholecystitis, the inflammation of the gallbladder is most commonly caused by the obstruction of the cystic duct by gallstone. Cholangitis is a bacterial infection that can occur when the common bile duct is blocked by gallstone. In our present study, we observed that the adjusted HR for PLAs in patients with T2DM was increased in individuals without gallstone, cholecystitis or cholangitis compared with in patients with PLA who did not have T2DM. T2DM is a strong risk for PLAs in patients without biliary tract disease.

Hepatic tuberculosis (TB) is uncommon and accounts for $<1 \%$ of all tuberculous infection (23). Although there is an increased risk of tuberculosis and tuberculous abscess (tuberculoma) just in diabetic patients. It is difficult to diagnose hepatic TB in absence of previous history of TB or concurrent pulmonary involvement. Patients might be asymptomatic or present with non-specific constitutional symptoms such as fever, fatigue, weight loss, and night sweats (24). On cross-sectional imaging, hepatic TB can be comprehensively classified into micronodular and macronodular forms (25). Macronodules are more than $2 \mathrm{~cm}$ in size. Single macronodular hepatic TB is therefore at higher risk of being misdiagnosed as a neoplastic lesion or liver abscess (26). In this study, hepatic TB has been excluded based on occurrence of PLAs (ICD-9-CM 572.0) at inpatient record.

To our knowledge, this is the first study to examine where DM medication increases PLA risk in patients with T2DM. We considered all commonly used DM medications on the Taiwanese market during the study period, including metformin, glyburide, glimepiride, gliclazide, glipizide, glitazones, DPP4i, and acarbose. 
TABLE 3 | HRs and adjusted HRs for PLA in the T2DM cohort according to DM medications.

\begin{tabular}{|c|c|c|c|c|c|}
\hline Variable & Event & PYs & Rate & HR (95\% Cl) & $\mathrm{aHR}^{\dagger}(95 \% \mathrm{Cl})$ \\
\hline \multicolumn{6}{|c|}{ Metformin } \\
\hline No & 81 & 107,634 & 7.53 & Ref & Ref \\
\hline Yes & 85 & 175,290 & 4.85 & $0.65(0.48-0.88)$ & $0.69(0.47-1.01)$ \\
\hline \multicolumn{6}{|c|}{ Glyburide } \\
\hline No & 111 & 204,240 & 5.43 & Ref & Ref \\
\hline No & 118 & 171,670 & 6.87 & Ref & Ref \\
\hline Yes & 48 & 111,254 & 4.31 & $0.64(0.46-0.89)$ & $0.80(0.54-1.18)$ \\
\hline \multicolumn{6}{|c|}{ Gliclazide } \\
\hline No & 109 & 183,498 & 5.94 & Ref & Ref \\
\hline Yes & 57 & 99,426 & 5.73 & $0.99(0.72-1.37)$ & $1.37(0.94-2.00)$ \\
\hline \multicolumn{6}{|l|}{ TZD } \\
\hline No & 146 & 226,616 & 6.44 & Ref & Ref \\
\hline Yes & 20 & 56,308 & 3.55 & $0.57(0.36-0.91)$ & $0.87(0.52-1.45)$ \\
\hline \multicolumn{6}{|l|}{ DPP4i } \\
\hline No & 166 & 249,575 & 6.65 & Ref & Ref \\
\hline Yes & 0 & 33,349 & 0 & - & - \\
\hline \multicolumn{6}{|c|}{ Acarbose } \\
\hline No & 151 & 227,523 & 6.64 & Ref & Ref \\
\hline Yes & 15 & 55,401 & 2.71 & $0.42(0.25-0.71)$ & $0.51(0.29-0.90)$ \\
\hline \multicolumn{6}{|l|}{ Others } \\
\hline No & 143 & 239,250 & 5.98 & Ref & Ref \\
\hline
\end{tabular}

${ }^{\dagger}$ Model adjusted for age, sex, gallstone, cholecystitis, cholangitis and each DM medications. PYs, person-years; Rate, incidence rate, per 10,000 person-years.

TZD, thiazolidinedione; DPP4i, Dipeptidyl peptidase-4 inhibitor.

In our analysis, we found that $60.4 \%$ of patients were prescribed metformin, $36.5 \%$ were prescribed glimepiride, $31.2 \%$ were prescribed gliclazide, $23.7 \%$ were prescribed glyburide; acarbose, glitazones, glipizide, DPP4i and other medications were prescribed at rates of $16.9,16.4,15.8,10.2$, and $13.8 \%$ respectively. Metformin was the most frequently prescribed DM medication. Compared with comparison cohort, the patients with T2DM prescribing glyburide exhibited a significantly increased of PLA risk, whereas acarbose is potentially associated with a reduced PLA risk in patients with T2DM.

Our study provided evidence that compared with other DM medications, acarbose reduces the PLA risk in patients with T2DM. One large nationwide, population-based cohort study in Taiwan showed that, in patients with DM who used acarbose, colorectal cancer risk was reduced by $27 \%$ compared for patients with DM not using acarbose (27). Acarbose is an $\alpha$-glucosidase inhibitors that delays the hydrolysis of disaccharides and polysaccharides to glucose in the small intestine. The mechanism for the prevention of colorectal cancer by acarbose is that acarbose increase more butyrate production by microbial fermentation after delayed carbohydrate absorption. Butyrate inhibits the growth of transformed cells and stimulates normal colonic mucosal proliferation (28). Another explanation for the reduction of colorectal cancer is that acarbose reduces colonic transit time and changes the fecal concentration of bile acids (29). A cohort study of 14,690 Taiwanese patients with PLA revealed an adjusted HR value for colorectal cancer of 5.5 for patients with PLA compared with controls (30). Therefore, acarbose may ostensibly reduce PLA risk in the newly diagnosed patients with T2DM.

Overall PLA incidence in the comparison cohort increase overtime, where the incidence in the DM cohort decreased from 4.02 in the first 2 years to 2.13 after 4 years of follow-up. The PLA occurrence in patient with DM was greatest at $0-2$ years followup $(\mathrm{HR}=4.02,95 \% \mathrm{CI}=2.87-5.63)$. PLA always accompanied newly or recently diagnosed T2DM with poor glycemic control.

The current study was a large, representative, nationwide, population-based sample to explore PLA risk in patients with newly diagnosed T2DM. However, it has few limitations. First, our study group excluded the individuals younger than 18 years. Second, we could not identify other potential 
TABLE 4 | Demographic factor and comorbidity-stratified analysis of HR for PLA risk in comparison and DM cohort.

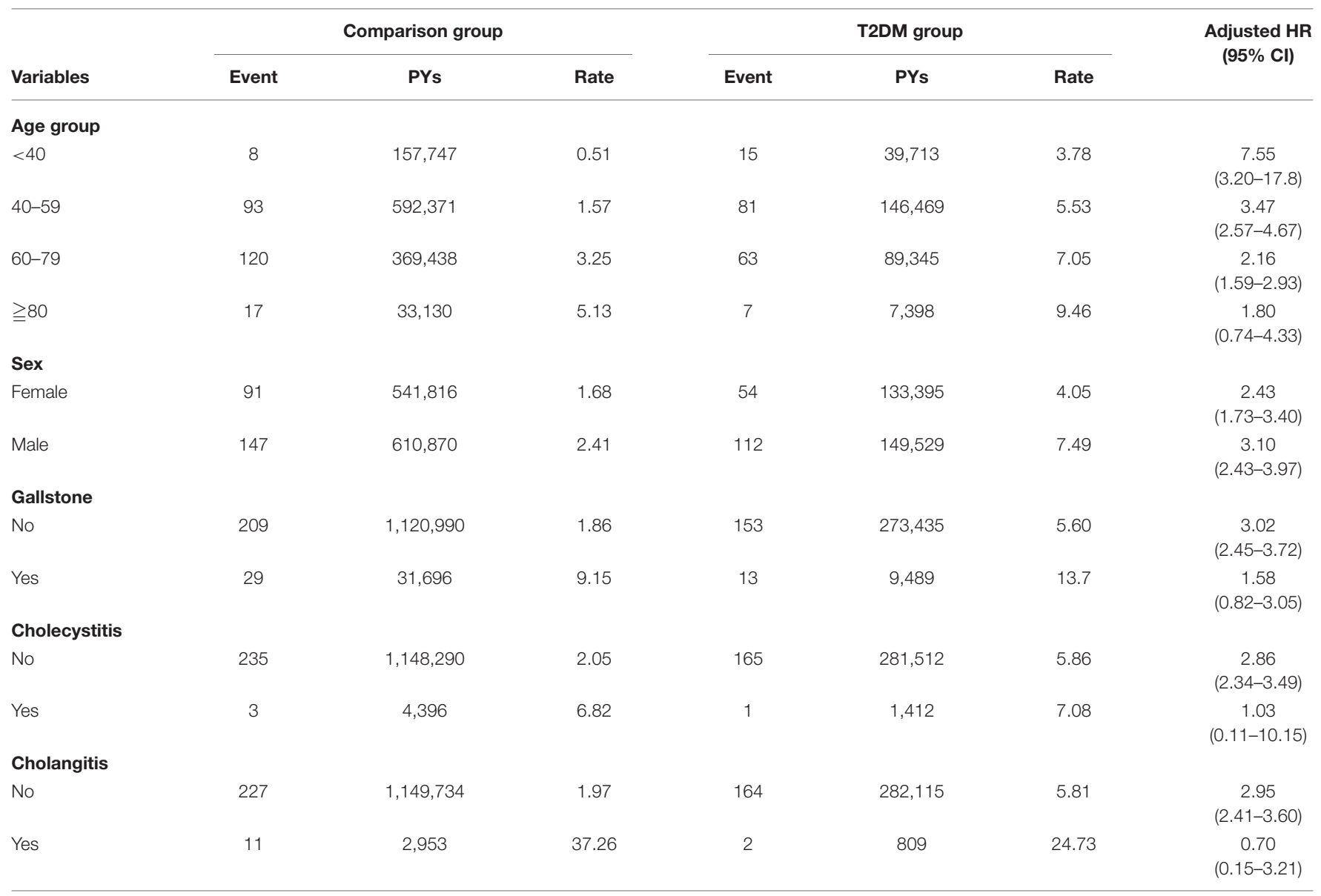

Model adjusted for age, sex, gallstone, cholecystitis, and cholangitis.

Rate, incidence rate, per 10,000 person-years.

TABLE 5 | HRS for PLA, according to follow-up duration.

Comparison cohort

Follow-up duration

Year <

Year 2-4

Year $\geqq 4$

Model adjusted for age, sex, gallstone, cholecystitis, and cholangitis.

PYs, person-years; Rate, incidence rate, per 10,000 person-years.

confounding factors in our database, such as smoking, alcohol consumption, nutritional status and environmental factors due to database limitation. Third, laboratory data such as blood glucose levels; hemoglobin A1c levels; liver function test results; imaging such as abdominal ultrasound or computed tomography; and blood cultures for KP, E. coli or other pathogens were unavailable. Other potential limitation to our study were selection bias or confounding by indication. Our selection bias and confounding by indication were patients with T1DM, age younger than 18 years old and other conditions such as poor immune conditions or medications for immune suppression.

In conclusion, PLA risk in patients with newly diagnosed T2DM was 2.83-fold higher than that in the comparison 
TABLE 6 | Microorganism test results of patients with PLA ( $N=404)$.

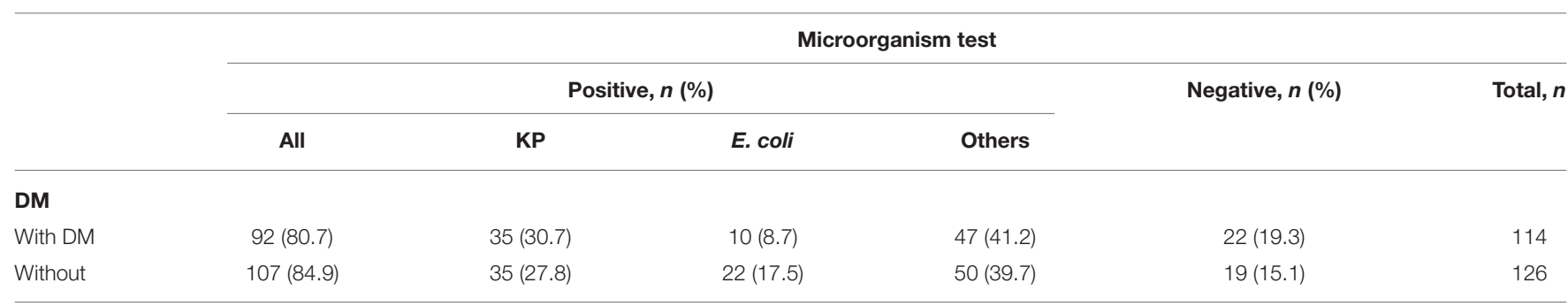

cohort. Younger patients and men with T2DM as well as patients with duration of DM $<2$ years had increased PLA risk. PLA risk was higher in newly diagnosed T2DM patients with gallstones and cholecystitis. In addition, compared with other DM medication, acarbose was associated with a lower PLA risk. Our findings highlight the value of active treatment strategies and close follow-up of patients with T2DM, and the effect of acarbose on PLA merits further investigation.

\section{DATA AVAILABILITY STATEMENT}

The datasets presented in this article are not readily available because the dataset used in this study is held by the Taiwan Ministry of Health and Welfare (MOHW). The Ministry of Health and Welfare must approve our application to access this data. Any researcher interested in accessing this dataset can submit an application form to the Ministry of Health and Welfare requesting access. Please contact the staff of MOHW (Email: stcarolwu@mohw.gov.tw) for further assistance. Taiwan Ministry of Health and Welfare Address: No.488, Sec. 6, Zhongxiao E. Rd., Nangang Dist., Taipei City 115, Taiwan (R.O.C.). Phone: +886-2-8590-6848. All relevant data are within the paper. Requests to access the datasets should be directed to Please contact the staff of MOHW (Email: stcarolwu@mohw.gov.tw) for further assistance.

\section{REFERENCES}

1. Jopsen P, Vilstrup H, Schonheyder HC, Sorensen HT. A nationwide study of the incidence and 30-days mortality rate of pyogenic liver abscess in Denmark, 1977-2002. Aliment Pharmacol Ther. (2005) 21:1185-8. doi: 10.1111/j.1365-2036.2005. 02487.x

2. Kaplan GG, Gregson DB, Laupland KB. Population-based study of the epidemiology of and the risk factors for pyogenic liver abscess. Clin Gastroenterol Hepatol. (2004) 2:1032-8. doi: 10.1016/S1542-3565(04)00459-8

3. Chen YC, Lin CH, Chang SN, Shi ZY. Epidemiology and clinical outcome of pyogenic liver abscess: an analysis from the National Health Insurance Research Database of Taiwan, 2000-2011. J Microbiol Immunol Infect. (2016) 49:646-53. doi: 10.1016/j.jmii.2014.08.028

4. Chen SC, Yen CH, Lai KC, Tsao SM, Cheng KS, Chen CC, et al. Pyogenic liver abscesses with Escherichia coli: etiology, clinical course, outcome, and prognostic factors. Wien Klin Wochenschr. (2005) 117:80915. doi: 10.1007/s00508-005-0481-1

\section{ETHICS STATEMENT}

The studies involving human participants were reviewed and approved by the Ethics Review Board of China Medical University (CMUH104-REC2-115-AR4). Written informed consent for participation was not required for this study in accordance with the national legislation and the institutional requirements.

\section{AUTHOR CONTRIBUTIONS}

C-HK: administrative support. All authors have contributed significantly and are in agreement with the content of the manuscript, conception and design, collection and assembly of data, data analysis and interpretation, manuscript writing, and final approval of manuscript.

\section{FUNDING}

This study was supported in part by Taiwan Ministry of Health and Welfare Clinical Trial Center (MOHW109-TDUB-212-114004), China Medical University Hospital (CMU107ASIA-19, DMR-109-231, DMR-110-089); MOST Clinical Trial Consortium for Stroke (MOST 108-2321-B-039-003-), TsengLien Lin Foundation, Taichung, Taiwan. The funders had no role in the study design, data collection and analysis, the decision to publish, or preparation of the manuscript.

5. Chang FY, Chou MY. Comparison of pyogenic liver abscesses caused by Klebsiella pneumoniae and non-K. pneumoniae pathogens. J Formos Med Assoc. (1995) 94:232-7.

6. Wang JH, Liu YC, Lee SS, Yen MY, Chen YS, Wang JH, et al. Primary liver abscess due to Klebsiella pneumoniae in Taiwan. Clin Infect Dis. (1998) 26:1434-8. doi: 10.1086/516369

7. Lin YT, Wang FD, Wu PF, Fung CP. Klebsiella pneumoniae liver abscess in diabetic patients: association of glycemic control with the clinical characteristics. BMC Infect Dis. (2013) 30;13:56. doi: 10.1186/1471-2334-13-56

8. Lin LY, Warren-Gash C, Smeeth L, Chen PC. Data resource profile: the National Health Insurance Research Database (NHIRD). Epidemiol Health. (2018) 40:e2018062. doi: 10.4178/epih.e2018062

9. Thomsen RW, Jepsen P, Sørensen HT. Diabetes mellitus and pyogenic liver abscess: risk and prognosis. Clin Infect Dis. (2007) 44:1194201. doi: $10.1086 / 513201$

10. Thomsen RW, Hundborg HH, Lervang HH, Johnsen SP, Schønheyder HC, Sørensen HT. Diabetes mellitus as a risk and prognostic factor for 
community-acquired bacteremia due to enterobacteria: a 10-year, populationbased study among adults. Clin Infect Dis. (2005) 40:628-31. doi: 10.1086/ 427699

11. Muller LM, Gorter KJ, Hak E, Goudzwaard WL, Schellevis FG, Hoepelman $\mathrm{AI}$, et al. Increased risk of common infections in patients with type 1 and type 2 diabetes mellitus. Clin Infect Dis. (2005) 41:281-8. doi: 10.1086/431587

12. Delamaire M, Maugendre D, Moreno M, Le Goff MC, Allannic H, Genetet B. Impaired leucocyte functions in diabetic patients. Diabet Med. (1997) 14:29-34. doi: 10.1002/(SICI)1096-9136(199701)14:1<29::AID-DIA300>3.0. $\mathrm{CO} ; 2-\mathrm{V}$

13. Hostetter MK. Handicaps to host defense. Effects of hyperglycemia on C3 and Candida albicans. Diabetes. (1990) 39:271-5. doi: 10.2337/diab.39.3.271

14. Lin YT, Liu CJ, Chen TJ, Fung CP. Long-term mortality of patients with septic ocular or central nervous system complications from pyogenic liver abscess: a population-based study. PLoS ONE. (2012) 7:e33978. doi: 10.1371/journal.pone.0033978

15. Cheng DL, Liu YC, Yen MY, Liu CY, Wang RS. Septic metastatic lesions of pyogenic liver abscess. Their association with Klebsiella pneumoniae bacteremia in diabetic patients. Arch Intern Med. (1991) 151:15579. doi: 10.1001/archinte.1991.00400080059010

16. Quah JH, Liu YP, Luo N, How CH, Tay EG. Younger adult type 2 diabetic patients have poorer glycaemic control: a cross-sectional study in a primary care setting in Singapore. BMC Endocr Disord. (2013) 13:18. doi: 10.1186/1472-6823-13-18

17. Song H, Wang X, Lian Y, Wan T. Analysis of the clinical characteristics of 202 patients with liver abscess associated with diabetes mellitus and biliary tract disease. $J$ Int Med Res. (2020) 48:300060520949404. doi: 10.1056/NEJM199912163 412507

18. Peleg AY, Weerarathna T, McCarthy JS, Davis TM. Common infections in diabetes: pathogenesis, management and relationship to glycaemic control. Diabetes Metab Res Rev. (2007) 23:3-13. doi: 10.1002/dmrr.682

19. Joshi N, Caputo GM, Weitekamp MR, Karchmer AW. Infections in patients with diabetes mellitus. N Engl J Med. (1999) 341:1906-12.

20. Chang FY, Chou MY, Fan RL, Shaio MF. A clinical study of Klebsiella liver abscess. Taiwan Yi Xue Hui Za Zhi. (1988) 87:282-7.

21. Frey CF, Zhu Y, Suzuki M, Isaji S. Liver abscesses. Surg Clin North Am. (1989) 69:259-71. doi: 10.1016/S0039-6109(16)44784-5

22. Casqueiro J, Casqueiro J, Alves C. Infections in patients with diabetes mellitus: a review of pathogenesis. Indian J Endocrinol Metab. (2012) 16(Suppl. 1):S2736. doi: $10.4103 / 2230-8210.94253$
23. Chong VH. Hepatobiliary tuberculosis: a review of presentations and outcomes. South Med J. (2008) 101:35661. doi: 10.1097/SMJ.0b013e318164ddbb

24. Forgione A, Tovoli F, Ravaioli M, Renzulli M, Vasuri F, Piscaglia F, et al. Contrast-enhanced ultrasound LI-RADS LR-5 in hepatic tuberculosis: case report and literature review of imaging features. Gastroenterol. Insights. (2021) 12:1-9. doi: 10.3390/gastroent 12010001

25. Pereira JM, Madureira AJ, Vieira A, Ramos I. Abdominal tuberculosis: imaging features. Eur J Radiol. (2005) 55:17380. doi: 10.1016/j.ejrad.2005.04.015

26. Kakkar C, Polnaya AM, Koteshwara P, Smiti S, Rajagopal KV, Arora A. Hepatic tuberculosis: a multimodality imaging review. Insights Imaging. (2015) 6:64758. doi: 10.1007/s13244-015-0440-y

27. Tseng YH, Tsan YT, Chan WC, Sheu WH, Chen PC. Use of an $\alpha$-glucosidase inhibitor and the risk of colorectal cancer in patients with diabetes: a nationwide, population-based cohort study. Diabetes Care. (2015) 38:206874. doi: $10.2337 / \mathrm{dc} 15-0563$

28. Weaver GA, Tangel CT, Krause JA, Parfitt MM, Stragand JJ, Jenkins PL, et al. Biomarkers of human colonic cell growth are influenced differently by a history of colonic neoplasia and the consumption of acarbose. J Nutr. (2000) 130:2718-25. doi: 10.1093/jn/130.11.2718

29. Ron Y, Wainstein J, Leibovitz A, Monastirsky N, Habot B, Avni Y, et al. The effect of acarbose on the colonic transit time of elderly long-term care patients with type 2 diabetes mellitus. J Gerontol A Biol Sci Med Sci. (2002) 57:M111-4. doi: 10.1093/gerona/57.2.M111

30. Lai HC, Lin CC, Cheng KS, Kao JT, Chou JW, Peng CY, et al. Increased incidence of gastrointestinal cancers among patients with pyogenic liver abscess: a population-based cohort study. Gastroenterology. (2014) 146:12937.e1. doi: 10.1053/j.gastro.2013.09.058

Conflict of Interest: The authors declare that the research was conducted in the absence of any commercial or financial relationships that could be construed as a potential conflict of interest.

Copyright (c) 2021 Wang, Lai, Chen, Wang, Hsieh, Chang, Chen, Ho, Hung, Tseng, Lin and Kao. This is an open-access article distributed under the terms of the Creative Commons Attribution License (CC BY). The use, distribution or reproduction in other forums is permitted, provided the original author(s) and the copyright owner(s) are credited and that the original publication in this journal is cited, in accordance with accepted academic practice. No use, distribution or reproduction is permitted which does not comply with these terms. 